\title{
Properties of kenaf filled unplasticized polyvinyl chloride composites.
}

\begin{abstract}
Combination of lignocellulosic fiber with thermoplastic is leading to the new areas of research in plastic composite field. Due to the problem of petroleum shortages and encouragement for reducing the dependence on fossil fuel products, thus increased the people interest in maximizing the utilize of renewable materials like kenaf fiber. By adding optimum natural fiber to thermoplastics could provide some cost reduction to the world of plastic industry as well as to dominance the agro-based industry. With a view to identifying the effect of fiber content and effect of coupling agent in kenaf fiber reinforced unplasticized poly (vinyl chloride) (UPVC) composite on the mechanical properties, the fiber and matrix mixture were mixed with poly [methylene poly (phenyl isocyanate)] (PMPPIC) using thermal mixing process followed by compression molding technique for the composite preparation that required for tensile characteristic (ASTM D638). The fiber loading were 10\%, 20\%, $30 \%$, and $40 \%$ in weight. Since the kenaf fiber and UPVC are chemically different, the compatibility and dispersability of kenaf fiber in UPVC can be improved by lowering the surface energy of the fiber to make it less polar, consequently more similar to the plastic matrix. Generally, PMPPIC act as a bonding agent that facilitates the optimum stress transfer at the interface between fiber and matrix which gives an optimal mechanical performance of kenaf fiber reinforced UPVC composites. Meanwhile, the addition of 30\% fiber contents with PMPPIC was successful to enhance the tensile properties and the efficiency of PMPPIC was verified using Fourier Transform Infra-Red (FTIR) spectroscopy.
\end{abstract}

Keyword: Coupling agent; FTIR; Kenaf; Lignocellulosic fiber; PMPPIC; PVC. 